\title{
Overview of Content-based Image Feature Extraction Methods
}

\author{
Lulu Fan, Zhonghu Yuan, Xiaowei Han, Wenwu Hua \\ College of Information Engineering University of Shenyang Shenyang, Liaoning Province, China \\ E-mail: 472221353@qq.com
}

\begin{abstract}
The technology of Image feature extraction, which is based on the subject of computer vision and image processing, use computer technology to determine the image of the invariant information and extract it to handle the actual problem. Based on the basic principle of image processing, the underlying characteristics of image and semantic feature extraction algorithm were introduced detailed in this paper.

Index Terms - Content-based; Feature Extraction; Image.
\end{abstract}

\section{Introduction}

As the rapid development of computer and network information technology, information extract has become has become crucial. It is very difficult to find the useful information manually in the vast ocean of information. Therefore, content-based image retrieval techniques have emerged.

There are two types in[1-3] of features which are used in content-based image retrieval technology, one type is the underlying characteristics or physical characteristics, another type is high-level features or semantic features, which is semantic description of the content of the image or logical link between various physical characteristics. The feature extraction algorithm of above characteristics was introduced detailedly in this paper based on the basic principle of image processing.

\section{Feature Extraction}

Feature extraction is a concept in computer vision and image processing. It refers to the image information extracted by using computer and to determine whether each image point belongs to a image characteristic. The result of Feature extraction is that the points on the image is divided into different subsets, these subsets tend to belong to the isolated points, continuous curve or continuous area ${ }^{[4]}$.

\section{Classification Of Feature Extraction Method}

\section{A. Color Feature}

Color feature is a global characteristic, it describes the surface properties of the scene in images or image area. General color feature is based on the characteristics of the pixel, That is to say all of the pixels belonging to image or image region has its own contribution.

1) Color Histogram: Color histogram in [3-7] is one of the most commonly method in color feature extraction. The color space need to be divided into several small range when Calculating color histogram, each interval become a handle (bin) of histograms. This process is called color quantization. In RGB color space, color histogram can be viewed as a 1d discrete functions:

$$
H(k)=\frac{n_{k}}{n}, k=0,1,2, \cdots L-1
$$

In the formula, $\mathrm{k}$ said image feature values, $\mathrm{L}$ said number of feature value which can be used, ${ }^{n_{k}}$ said number of pixel values which specific feature value is $\mathrm{k}, \mathrm{n}$ said the total number of image pixels.

2) Color Set: The color set in[8] is put forward by Smith and Chang. Color set is a kind of approximation for color histogram, first of all, the image must be changed from RGB color space into a visual balance color space (e.g., HSV space), and the color space is quantized into a handle. Then, using color image automatic segmentation technology to divide image into several regions, each region is indexed by using a certain color component in quantified color space, so that the image will be expressed as a binary image color index set.

3) Color Moment: Color moment in[8] is a simple and effective characteristic, is put forward by Stricker and Oreng, the mathematical foundation of this method is any color distribution of the image can be represented by its moment. In addition, because the information of color distribution is mainly concentrated in the low moments, only using scheme, the Variance and the Skewness of the color is enough to express the image of the color distribution, compared with the color histogram, another benefit of this approach is not need to quantify the characteristics.

\section{B. Texture Feature}

Texture feature in[9-12] is the intrinsic characteristics that are shared by all objects surface, and contains important information about the organization structure on the surface of the object and their links with the surrounding environment. The main purpose of texture feature extraction is turning differences in the space of the random texture or geometric structure into differences in characteristics of grey value, describing the image texture information with some mathematical model, including smooth image area, sparse, regularity and so on. Extraction method can be divided into statistical method, structure method and spectrum method, etc.

1) Statistical Methods: Texture characteristics, especially the natural texture, show a lot of randomness in the local analysis, most can be described as a random variable. But from 
the overall analysis and statistical sense, it also exists some regularity. Method based on statistics in[13] are gray histogram moments, autocorrelation function, analysis of gray level co-occurrence matrix, fringe frequency and primitive stroke length, etc. Below is a simple introduction for two methods.

Feature extraction based on image gray histogram in[14]:

(1) Converting color images to grayscale image.

(2) Taking down the number of occurrences of various gray scale images in gray histogram, getting gray histogram, calculating the mean gray value.

(3) Calculating feature vector.

Calculating $\mathrm{n}$ moments of histogram $(\mathrm{n}=1,2,3,4)$ With moments formula, the texture feature of images can be described with the four feature vector.

The feature extraction based on histogram of pixel-value differences in[14]:

(1) Converting color images to grayscale image.

(2) Calculating pixel-value differences of adjacent pixels in the image. For the edge pixels, calculating the pixel-value difference between pixel and its 8 pixels within $3 \times 3$ neighborhood; For edge pixels, the number of the neighbor hood varies with the location.

(3) Calculating histogram of pixel-value differences. If range of pixel-value differences is $0 \sim 255$, doing the statistics of the number of occurrences in each gray level to get histogram of pixel-value differences.

(4) Textural features can be described by contrast, second moment of angle direction, entropy and the average:

$$
\begin{aligned}
& C_{o n}=\sum_{h=0}^{L} h^{2} p(h) \\
& A_{s m}=\sum_{h=0}^{L} p^{2}(h) \\
& E_{n t}=-\sum_{h=0}^{L} p(h) \log _{2} p(h) \\
& M_{\text {ean }}=\frac{1}{L} \sum_{h=0}^{L} h-p(h)
\end{aligned}
$$

In the formulas $\mathrm{h}$ is the pixel-value differences, $\mathrm{p}(\mathrm{h})$ is probability of $\mathrm{h}$.

$C_{o n} 、 A_{s m} 、 E_{n t}$ and $M_{\text {ean }}$ can be gotten by using formulas (2) $\cdot(3) \cdot(4)$ and (5).

2) Structure Approach: Primitive shape and the distribution features are analysed by method of the structure approach in angle of the result of the texture image texture, the basic idea is that complex texture can be made of some simple primitives in the form of a regular combination. Area, perimeter, degree of eccentricity, direction, extent, euler number, moment and amplitude characteristics are the feature of texture primitive, if some primitive rules can be defined, texture pattern required is constituted with the relevant provisions of the texture primitive.
3) Spectrum Methods: Texture image is transformed into frequency domain with spectrum method in[15], the texture feature such as cycle, power spectrum, etc are gotten by using signal processing method in the space domain. Fourier transform and wavelet transform are used common- ly.

The Fourier Transform: For a given image, a twodimensional Fourier transform can obviously includes all its texture information. Therefore, as texture feature is derived from the object itself, deriving from the frequency spectrum is also very useful. Directivity of the periodic or almost periodic two dimensional image is described By means of the frequency spectrum of Fourier spectrum.

The Wavelet Transform: Using wavelet transform in[1618] the energy of the original image can be concentrated to a small number of wavelet coefficients, and after the decomposition of wavelet coefficients the direction of the 3 detail component have high local correlation, which provides favorable conditions for feature extraction.

Texture feature extraction algorithm based on wavelet transform is as follows:

(1) Converting color images to grayscale image.

(2) Wavelet decomposition of four layers.

(3) Calculating the mean and standard deviation after wavelet decomposition. Set the subband after wavelet deco- mposetion is $f_{i}(x, y)(M \times N), i=1,2, \cdots 13$ :

$$
\begin{aligned}
u_{i} & =\frac{1}{M \times N} \sum_{j=1}^{M} \sum_{k=1}^{N}\left|f_{i}(x, y)\right| \\
\sigma_{i} & =\sqrt{\frac{\sum_{j=1}^{M} \sum_{k=1}^{N}\left(\left|f_{i}(x, y)\right|-u_{i}\right)^{2}}{M \times N}}
\end{aligned}
$$

(4) Obtaining eigenvector $\mathrm{T}$ : taking the mean and standard deviation of each subband of wavelet coefficient as each component of image texture feature vector, eigenvector $T=\left\lfloor u_{1}, \sigma_{1}, u_{2}, \sigma_{2, \ldots} u_{13}, \sigma_{13}\right\rfloor$;

(5) The normalized processing of feature: due to the differences of the physical meaning and the scope among 13 feature vectors, it need to be normalized in case of large deviation. Taking gaussian normalization as example, after normalization a few of large or super small element have little effect on the normalized value points. If the original feature vector is $\left[t_{1}, t_{2}, \cdots t_{N}\right]$, calculating the mean and standard deviation $\sigma$, if the normalized vector is $\left[T_{1}, T_{2}, \cdots T_{N}\right]$ :

$$
T_{i}=\frac{t_{i}-m}{\sigma}
$$

After normalization with formula (8), every component will be disposed to the domain[-1,1].

\section{Shape Feature}

Since the same object may have a variety of different colors but similar shape, a lot of queries may not be aimed at the color of the image. Generally speaking, there are two kinds of methods to present shape features, one is describing their 
contour feature, and another is regional characteristics. Therefore, image feature extraction method based on shape should also be divided into feature extraction that is based on contour (boundary) and feature extraction based on region.

\section{1) Feature Extraction Based On Contour}

Simple Descriptor: (1) Contour perimeter. The contour circumference refers to the minimum length of the outer boundary in the whole connected domain. We can indicate its length by the number of pixel on the contour; also can we calculate its circumference by chain code method.

(2) Contour diameter. Contour diameter is the furthest distance between two points on the contour. The diameter of the contour Y may be defined as:

$$
d(Y)=\max _{i j}\left\lfloor d\left(p_{i}, p_{j}\right)\right\rfloor
$$

$\mathrm{d}$ can be Euclidean distance or other distance, $p_{i}$ and $p_{j}$ are the points on the contour.

(3) Eccentricity. The long axis is the ligature (straight line) of two endpoints on the contour diameter. The short axis can be defined as longest straight line that is perpendicular to the long axis and intersected with contour boundary. The ratio of the long axis and the short axis is the contour of the eccentricity.

Fourier Descriptor: Fourier descriptor is one of the important methods to analysis and identifies object shape. It uses continuous Fourier transform method, which is based on the curve polygonal approximation, to calculate the Fourier descriptor, and eliminates phase influence of starting point on the boundary by the shape of the main direction. Fourier descriptor is Fourier transform coefficients of object shape boundary curve, it is the results of analysis signals of object boundary curve. According to the properties of Fourier transform, Fourier descriptor is associated with the scale of the shape, direction and the starting point position of the curve. Therefore we need to normalize Fourier descriptor which has the feature of rotation, translation and scaling invariance to identify the shape of the object.

Statistical Moment: Torque is a kind of statistical form of image pixels, so called statistical moment. The shape of contour can be rational described by the mean value, variance and the high order moment, etc.

\section{2) Shape Feature Extraction Based On The Area}

Simple Description: (1) The region area. Area of zone in Digital image is the number of pixels in the area. Pixel in a region is marked $f(x, y)=1$, outside the area is marked $f(x, y)=0$.The area can be expressed by the number of 1, for example: $S=\sum_{(x, y) \in R} 1$.

(2) Circular degree. Circular degree is used to describe level of similarity between the area shape and round shape. The area of the region and the contour length are often used as a descriptor under the condition of invariable target area size. For a certain area, the smaller the perimeter of regional outline, the more similarity with roundness, or it will be complex and rough. As to uncertain area of the image, the greater the density and the longer the perimeter of unit area, the more complex and changeable it will be. Circular measure the degree of target area tending to round, the formula is:

$$
C=\frac{p^{2}}{4 \pi A}
$$

In this formula $\mathrm{p}$ is the regional circumference; $\mathrm{A}$ is the area of the region. If area is circular, $\mathrm{C}$ will be taken the minimum value 1 , and when the region form into thin strips, circular degree $\mathrm{C}$ will be bigger.

(3) Regional center of gravity. the formula of mass center:

$$
\bar{x}=\frac{1}{A} \sum_{(x, y) \in R} x \quad, \quad \bar{y}=\frac{1}{A} \sum_{(x, y) \in R} y
$$

$(\mathrm{x}, \mathrm{y})$ is a point in the area, $\mathrm{A}$ is regional area.

Topology Descriptor: Topological feature is very useful for the overall description of the image in plane domain. Common topology description contains: number of connected components $\mathrm{C}$, regional void number $\mathrm{H}$ and Euler number $\mathrm{E}$.

$$
\mathrm{E}=\mathrm{C}-\mathrm{H}
$$

These characteristic calculations are not only relatively simple but also have the features of shift invariance, rotation invariance and scale invariance.

\section{Spatial Features}

Spatial relations in refers to the relationship of space position or relative direction relations between multiple target that are formed by image segmentation, which can also be divided into adjacency relationship, overlapping and inclusion relation, etc. Usually space position information can be divided into two categories: relative space position information and the absolute space location information. The former relation stresses the relative situation between targets. The latter stress the distance and orientation between the targets.

There are two kinds of commonly used extraction methods, one of which is extracting features after image segmentation according to the image or color and other characteristics of objects, another is to extracted the feather of each sub-block image that are regularly segmented.

\section{E. High-level Features Or Semantic Features}

Actually semantic feature extracting includes compatible semantic feature extracting and distinctive semantic features extracting. Extraction ways can be roughly divided into three kinds: (1) Compatible semantic feature extraction can be extracted by words assemble, meaning of the words together and paraphrase from dictionary. (2) Distinctive semantic features extracting can be achieved by the syntactic transformation, property contrast or add a component, etc. (3) the integrated use of semantic feature extraction method. Due to the restrictions of semantic features to combination and aggregation and even the syntactic properties of the words, the analysis of semantic feature sometimes integrated use a variety 
of methods from different angles, instead of confining to a particular method.

\section{Comparison Of Feature Extraction Method}

TABLE I. COMPARISON OF FEATURE EXTRACTION METHOD

\begin{tabular}{|c|c|c|c|}
\hline \multirow{2}{*}{$\begin{array}{c}\text { Feature } \\
\text { extraction } \\
\text { method }\end{array}$} & \multicolumn{3}{|c|}{ Comparison of feature extraction method } \\
\hline & Advantages & Disadvantages & Scope of application \\
\hline $\begin{array}{c}\text { Color } \\
\text { feature } \\
\text { extraction }\end{array}$ & $\begin{array}{l}\text { Simple, } \\
\text { Global of } \\
\text { color } \\
\text { description } \\
\text { is good. }\end{array}$ & $\begin{array}{l}\text { Cannot describe } \\
\text { local } \\
\text { distribution, } \\
\text { unable to } \\
\text { describe the } \\
\text { color spatial } \\
\text { location,cannot } \\
\text { describe the } \\
\text { specific object. }\end{array}$ & $\begin{array}{l}\text { Difficult image } \\
\text { segmentation, } \\
\text { regardless of the } \\
\text { image space } \\
\text { distribution. }\end{array}$ \\
\hline $\begin{array}{c}\text { Texture } \\
\text { feature } \\
\text { extraction }\end{array}$ & $\begin{array}{l}\text { Global, } \\
\text { stability, } \\
\text { rotation } \\
\text { invariance, } \\
\text { anti-noise. }\end{array}$ & $\begin{array}{l}\text { Relatively } \\
\text { complicated, } \\
\text { time } \\
\text { consuming. }\end{array}$ & $\begin{array}{l}\text { Visually } \\
\text { distinguishable } \\
\text { texture image, does } \\
\text { not apply to a } \\
\text { variety of texture } \\
\text { types of complicated } \\
\text { boundary problem. }\end{array}$ \\
\hline $\begin{array}{c}\text { Shape } \\
\text { feature } \\
\text { extraction }\end{array}$ & $\begin{array}{l}\text { Intuitive, } \\
\text { easy to } \\
\text { understand, } \\
\text { ability to } \\
\text { identify the } \\
\text { target. }\end{array}$ & $\begin{array}{l}\text { The lack of } \\
\text { model, high } \\
\text { requirements } \\
\text { about } \\
\text { computation } \\
\text { and storage. }\end{array}$ & $\begin{array}{l}\text { The target shape } \\
\text { does not change. }\end{array}$ \\
\hline $\begin{array}{c}\text { Spatial } \\
\text { feature } \\
\text { extraction }\end{array}$ & $\begin{array}{l}\text { Strengthen } \\
\text { the ability } \\
\text { to } \\
\text { distinguish } \\
\text { between } \\
\text { image } \\
\text { description. }\end{array}$ & $\begin{array}{l}\text { Sensitive to } \\
\text { rotate, inversion } \\
\text { and scale } \\
\text { changes }\end{array}$ & $\begin{array}{l}\text { Used in combination } \\
\text { with other methods. }\end{array}$ \\
\hline $\begin{array}{l}\text { Semantic } \\
\text { feature } \\
\text { extraction }\end{array}$ & $\begin{array}{l}\text { Accurate } \\
\text { description } \\
\text { of the } \\
\text { image }\end{array}$ & $\begin{array}{l}\text { Complex } \\
\text { difficult }\end{array}$ & $\begin{array}{l}\text { The method is in the } \\
\text { theoretical } \\
\text { assumptions and } \\
\text { exploration stage. }\end{array}$ \\
\hline
\end{tabular}

\section{Conclusions}

In image retrieval technique based on content, image feature extraction is essential, technology of which also has been paid more and more attention from scholars. But there are still inadequate such as relatively complex calculation and the lack of a unified evaluation criterion, etc. Improving classical algorithms and putting forward a new theory method become direction for scholars to strive for. Integration of many feature extraction methods become the scholar's research direction, since single feature is difficult to accurately express the accuracy of image information.

\section{References}

[1] Xianglin Huang, Lansun Shen,"The technology research of contentbased image retrieval," Journal of electronics, vol.30, no.7, pp.10651071, July 2002.

[2] Qingqi Hong,"Study and implementation of content based image retrieval,"Xiamen,China: Xiamen University,2005

[3] Jixiang Sun, The image processing,Beijing: Science press, 2010.

[4] Yan Hang, Yubin Yang, Zhaoqian Chen,"Review of content based image retrieval,"Computer application research,vol.19,no.9,pp.9-13,sep 2002.

[5] David A White Similarity Indexing: Algorithms and Pe-rformance 1996

[6] Selem Aksoy,Robert M,"Haralick Feature Normalization and Likelihood-based Similarity Measures for Image Retrieval," vol.22, 2 001

[7] FENG H Y,PAVLIDIS T, "Dccomposition of Polygons Into Simpler Components:Feature Extraction for Syntactic Pattern Recogntion," IEEE Transactions on Computers,vol.24,pp.636-650, 1975

[8] Stricker MA,Orengo M, "Similarity of color images,"in : Proc. of SPIE : Storage and Retrieval for image and Video Databases III, vol.2185,pp.381-392,Feb 1995.

[9] Li Liu,Gangyao Kuang,"Review of Image texture feature extraction methods," Chinese journal of image and graphics,vol.14,no.4,pp.622635,Apr 2009

[10] ChanghuaTang,"Research of content-based image retrieval method," Changchun,China: Northeast Normal University,2007

[11] Zilin Song, Zhibin Lu, "Algorithm research of Content based image feature extraction,"Paper presented at the tenth academic annual conference of the jiangsu Association for System engineering,2007

[12] Liangshen Wang,"Research of Image feature extraction and contentbased image database retrieval theory,"Dalian,China:Dalian Univer- sit $\mathrm{y}$ of Technology,2002

[13] Huiming Wang,Ping Shi,"Method of Image texture feature extraction,"Journal of communication university of China natural science, vol.13,no.1,pp.49-52,Jan 2006.

[14] Chang T,Jay Kuo C C,"Texture analysis and classification with truestructured wavelet transform,"IEEE Trans on Image Processing, vol. 2,no.4,pp.429-441,October 1993.

[15] Laine A,Fan J,“Texture classification by wavelet packet signatures," IEEE Trans Pattern Analysis and Machine Intelligence,vol.15, no. 11,pp.1186-1191,Nov 1993.

[16] Bingchun Li, “Feature extraction of image zoom edge shape,"Journal of Kashgar teachers college,vol.28,no.3,pp.62-64,May 2007.

[17] Tao Wang,Shimin Hu,Jiaguang Sun, "Image retrieval based on color space characteristics,"Journal of software, vol.13,no.10,pp.20312036,Jun 2002.

[18] Changxi Rui,"Function and semantic feature extraction of semantic feature analysis ,"The northern review,vol.3,pp.66-70,2005. 